\title{
Defenders against overdiagnosis
}

\author{
Richard Hurley features and debates editor
}

The BMJ

There's money to be made in diagnosis. Consider the buzz around immunosignature tests for antibodies to diagnose cancers or infections. Many other fledgling diagnostic technologies (breath testing for lung cancer, patches for vital sign monitoring, biomarkers for Alzheimer's disease) have also shown enough clinical promise to seduce the capital investors.

Indeed, global revenues in the mobile health monitoring market are expected to grow 10-fold between 2012 and 2018, to £17bn (€19.4bn; \$22.2bn), and the annual global biomarker market is expected to reach $£ 80$ bn by 2020 .

One hope is that all this interest in new diagnostic technologies will transform medicine, making it more health promoting, more proactive, and more personalised, perhaps in the ways our recent essayist Michael Millenson described (doi:10.1136/bmj.j3048).

But new tests also risk labelling more healthy people as sick, and false positives will lead to overdiagnosis and overtreatment, with the potential for physical and mental harm. So say Bjørn Hofmann and H Gilbert Welch in an Analysis article this week (doi:10.1136/bmj.j3314). False alarms and indeterminate findings will worry patients, drive more testing, increase clinical workload, and distract clinicians from more important work.
And then there's the profit motive, encouraging unnecessary testing in the first place, they warn. "Promotional campaigns will necessarily need to get people concerned about disease and indicate that the path to health is through testing-reinforcing health anxiety in some and distracting many from more important health behaviours," they say.

Ideally, all new diagnostic tests would be subject to rigorous, independent assessment, they say. "Researchers should try to answer three questions. Firstly, does the test reliably predict a health event that matters to patients? Secondly, can that risk be lowered by an effective action? Many tests will fail here, but for those that don't, the final question is, what happens to those who do not benefit?"

As for doctors, they have an essential role in protecting people from the harms of new diagnostic tools, say the authors. They can provide this "strong counterbalance" in various ways: by ensuring that their patients fully understand the harms of testing as well as the possible benefits; by not testing people at low risk; by thinking through all the possible implications of testing; and being prepared for overly positive feedback about the use of new tests.

In these ways, there is money to be saved as well. 